Alfarama Journal of Basic \& Applied Sciences

Faculty of Science Port Said University

January 2021, Volume 2, Issue 1 https://ajbas.journals.ekb.eg ajbas@sci.psu.edu.eg

http://sci.psu.edu.eg/en/

DOI: $10.21608 /$ ajbas.2020.32855.1019

Submitted: 21-06-2020

Accepted: 26-07-2020

Pages: 51-59

\title{
Role of Omentin-1 in the Diagnosis and Prognosis of Breast Cancer in Egyptian
} Females.

\author{
F. Z. Mohammed ${ }^{1}$, Lamis Gamal ${ }^{2, *}$, Mohamed Farouk Mosa ${ }^{3}$, Mohamed Ibraheim Aref ${ }^{3}$. \\ ${ }^{1}$ Department of Biochemistry, Faculty of Science, Zagazig University, Egypt. \\ ${ }^{2}$ Department of Biochemistry, Faculty of Science, Port Said University, Egypt. \\ ${ }^{3}$ Department of Clinical Pathology, Faculty of Medicine, Al-Azhar University, Egypt. \\ *Corresponding author: dr.lamis_gamal@yahoo.com
}

\begin{abstract}
Background: in Egypt, Breast carcinoma is the most common malignancy affecting women. It is claimed for $32 \%$ of cancer in women. It is essential to clarify and assess prognostic factors associated with the breast cancer as they are the corner stone to identify the high risk groups who can benefit from adjuvant therapy and improving their disease prognosis. Aim of the study: in this study, we aimed to assess the value of serum omentin-1 levels as prognostic markers for breast cancer in Egyptian females. Patients and methods: 120 BC patients and 30 age and BMI matching health controls are the subjects of the study; we assessed the omentin-1 levels in the serum and correlated them with the clinicopathologic aspects in the BC patients. Results: our study showed that omentin-1 is significantly differing between healthy controls and BC patients, and revealed gradual increase with disease severity. Conclusion: our findings suggested that omentin-1 could be diagnostic marker for early cases detection and associated with breast cancer prognosis, supporting its role as prognostic biomarkers.
\end{abstract}

ABSTRACT

\section{Keywords}

Breast cancer, diagnosis, prognosis, omentin-1.

\section{Abberviation}

BC:breast cancer BMI:body mass index National Cancer Institute-Cairo University (NCI-CU ELISA :enzyme linked immunosorbent assay NPI: The Nottingham prognostic index (NPI)

\section{INTRODUCTION}

Breast cancer is a disease well characterized by etiological and pathological heterogeneity, it shows extreme variability in the course and prognosis of affected patients. Much effort on the scientific and organizational levels are aiming towards better understanding of the etiological and molecular motivations controlling breast cancer disease onset and prognosis as well as the best intervention lines with the best benefits and the least hazards [1]. 
In Egypt, Breast carcinoma is the most common malignancy affecting women. It is claimed for $32 \%$ of cancer in women. In the National Cancer Institute-Cairo University (NCI-CU), breast cancer cases are representing $19.3 \%$ of total primary malignancies [2]. These data is giving indicators about the extent of the disease as well as its morbidity and mortality magnitude, eliciting the urgent need for proper management protocols [3].

For many malignancies, serum tumor markers play an important role in patient management). In breast cancer, however, the role of serum markers is less well established. The most widely used serum markers in breast cancer are CA 15-3 and carcinoembryonic antigen (CEA). Less widely used markers include BR 27.29 (also known as CA27.29), tissue polypeptide antigen (TPA), tissue polypeptide specific antigen (TPS) and the shed form of HER-2. The potential uses of serum markers in breast cancer include aiding early diagnosis, determining prognosis, prospectively predicting response or resistance to specific therapies, surveillance after primary surgery, and monitoring therapy in patients with advanced disease. The aim of this review is to examine the role of serum tumor markers in the detection and management of patients with breast cancer [4].

The American Joint Committee on Cancer (AJCC) has designated staging by TNM classification to define breast cancer (table 2). The TNM system is based on the size and/or extent (reach) of the primary tumor (T), the amount of spread to nearby lymph nodes $(\mathrm{N})$, and the presence of metastasis $(\mathrm{M})$ or secondary tumors formed by the spread of cancer cells to other parts of the body. A number is added to each letter to indicate the size and/or extent of the primary tumor and the degree of cancer spread [5].

Accordingly, one adipokine that is secreted from visceral fat, placenta and ovaries, is called omentin-1. It was first discovered by Yang et al. it was found that omentin-1 expression is decreased in cases of obesity and glucose metabolism disorders as impaired glucose orlerance, insulin resistance and type II diabetes mellitus as well as polycystic ovary syndrome in obese females [6].

Omentin has a molecular mass of $34 \mathrm{kDa}$, and it was first discovered in 2001 while plays a substantial role in defense mechanisms in the intestinal against harmful bacteria. There are two types of omentin, omentin-1 and omentin-2. Although omentin-1 is the most circulating form of omentin in blood, there is some evidence that serum omentin-1 level is decreased in obesity and its related disease such as insulin resistance and type 2 diabetes[7]

The association between obesity and cancer has been reported by several studies. Although the exact mechanism is unclear but adipocytokines such as omentin-1 considered as one possible mechanism linking obesity and cancer. Measurement of their serum levels may be early diagnostic markers for several types of cancer [8].

Omentin-1 is well known by its anti-inflammatory role in the vessels myocytes. Yet, variation in its levels secondary to variable types of cancers, either up or down regulations, showed conflicting results [9]. It was concluded omentin-1 has a principal effect in the regulation of pathogenesis and apoptotic process of cancer cells [10].

In this study, we aimed to assess the value of serum omentin-1 as biomarker in Egyptian females with breast cancer. 


\section{SUBJECTS AND METHODS}

\section{Patients}

This study is a case control study that was carried out in Zagazig university hospital, Egypt. It was performed in the period from January 2019 to January 2020. Zagazig university ethical committee approved the study.

Inclusion creiteria: female patients with breast cancer that where recently proved breast cancer of different disease stages

Exclustion creiteria: any patient with other malignancy (benign or malignant tumor In GIT, other chronic disease, autoimmunodiseaseand imflmmatory disorders like crohns disease)

- Group 1 (control): 30 healthy female subjects, age and BMI matching to the patient group.

- Group 2 (patients): 120 breast cancer patients that were, with recently pathologically proved breast cancer, of different disease stages.

\section{Methods}

Study subjects serum samples, that were -20 were assayed for serum omentin-1 levels guided by the steps of manufacturer's instructions (Biovendor Inc,Brno, Czech Republic).

Omentin-1 was based on standard sandwich enzyme-linked immune-sorbent assay technology (ELISA). - In the BioVendor Human Omentin-1 ELISA, standards, quality controls and samples were incubated at $37^{\circ} \mathrm{C}$ in microplate wells pre-coated with polyclonal anti-human omentin-1 antibody. After 120 minutes incubation and washing, biotin labelled polyclonal antihuman omentin-1 antibody was added and incubated at $37^{\circ} \mathrm{C}$ with captured omentin-1 for 30 minutes. - After another washing, streptavidin-HRP conjugate was added. After 30 minutes incubation at $37^{\circ} \mathrm{C}$ and the last washing step, the remaining conjugate was allowed to react with the substrate solution (TMB). - The reaction was stopped by addition of acidic solution and absorbance of the resulting yellow product is measured. The absorbance was proportional to the concentration of omentin-1. - A standard curve was constructed by plotting absorbance values against concentrations of standards, and concentrations of unknown samples are determined using standard curve.

\section{Assay procedure}

- $100 \mu \mathrm{l}$ of diluted Standards, Quality Controls, Dilution Buffer (=Blank) and samples were introduced in duplicates, into the appropriate wells. - The plate was incubated at room temperature $\left(25^{\circ} \mathrm{C}\right)$ for 1 hour, shaking was carried out at ca. $300 \mathrm{rpm}$ on an orbital microplate shaker. - The wells werewashed 3-times with Wash Solution ( $0.35 \mathrm{ml}$ per well). After final wash, the plate was inverted and tapped strongly against paper towel. - $100 \mu 1$ of Biotin Labelled Antibody solution was added into each well. The plate was incubated at room temperature $\left(25^{\circ} \mathrm{C}\right)$ for 1 hour, shaking was carried out at ca. $300 \mathrm{rpm}$ on an orbital microplate shaker. - The wells were washed 3-times with Wash Solution $(0.35 \mathrm{ml}$ per well). After final wash, the plate was inverted and tapped strongly against paper towel. - $100 \mu \mathrm{l}$ of Streptavidin-HRP Conjugate was added into each well. Patients and Methods $\square 87 \square$ - The plate was incubated at room temperature $\left(25^{\circ} \mathrm{C}\right)$ for 30 minutes, shaking was carried out at ca. $300 \mathrm{rpm}$ on an orbital microplate shaker. - The wells were washed 3-times with Wash Solution ( $0.35 \mathrm{ml}$ per well). After final wash, the plate was inverted and tapped strongly against paper towel. - $100 \mu 1$ of Substrate Solution was added into each well. - Incubate the plate for 10 minutes at room temperature. - The color development was stopped by adding $100 \mu \mathrm{l}$ of Stop Solution. - The absorbance of each well was determined using a microplate reader set to $450 \mathrm{~nm}$ (acceptable range: 550 - $650 \mathrm{~nm}$ ). Readings at 630 $\mathrm{nm}(550-650 \mathrm{~nm})$ was substracted from the readings at $450 \mathrm{~nm}$. 


\section{Statistical analysis}

MedCal_version 17.9.7 software was used for the analysis of the (MedCalc Software bib, Ostend, Belgium). Quantitave data were presented in the form of mean and standard deviation, while qualitative data were presented in the form of frequency and percentage. Nottingham prognostic index (NPI) values of the patients were calculated and interpreted [11]. Pearson tests were carried out for correlation of the serum marker with the clinic-pathological data of the patients. ROC curve analysis was done to estimate cutoff point for differentiation between healthy subjects and breast cancer patients.

\section{STATISTICAL ANALYSIS}

MedCal_version 17.9.7 software was used for the analysis of the (MedCalc Software bib, Ostend, Belgium). Quantitave data were presented in the form of mean and standard deviation, while qualitative data were presented in the form of frequency and percentage. Nottingham prognostic index (NPI) values of the patients were calculated and interpreted [11]. Pearson tests were carried out for correlation of the serum marker with the clinic-pathological data of the patients. ROC curve analysis was done to estimate cutoff point for differentiation between healthy subjects and breast cancer patients.

\section{RESULTS AND DISCUSSION}

Age and BMI (table 1)

Table 1: Mean \pm SD of women age and BMI among studied groups.

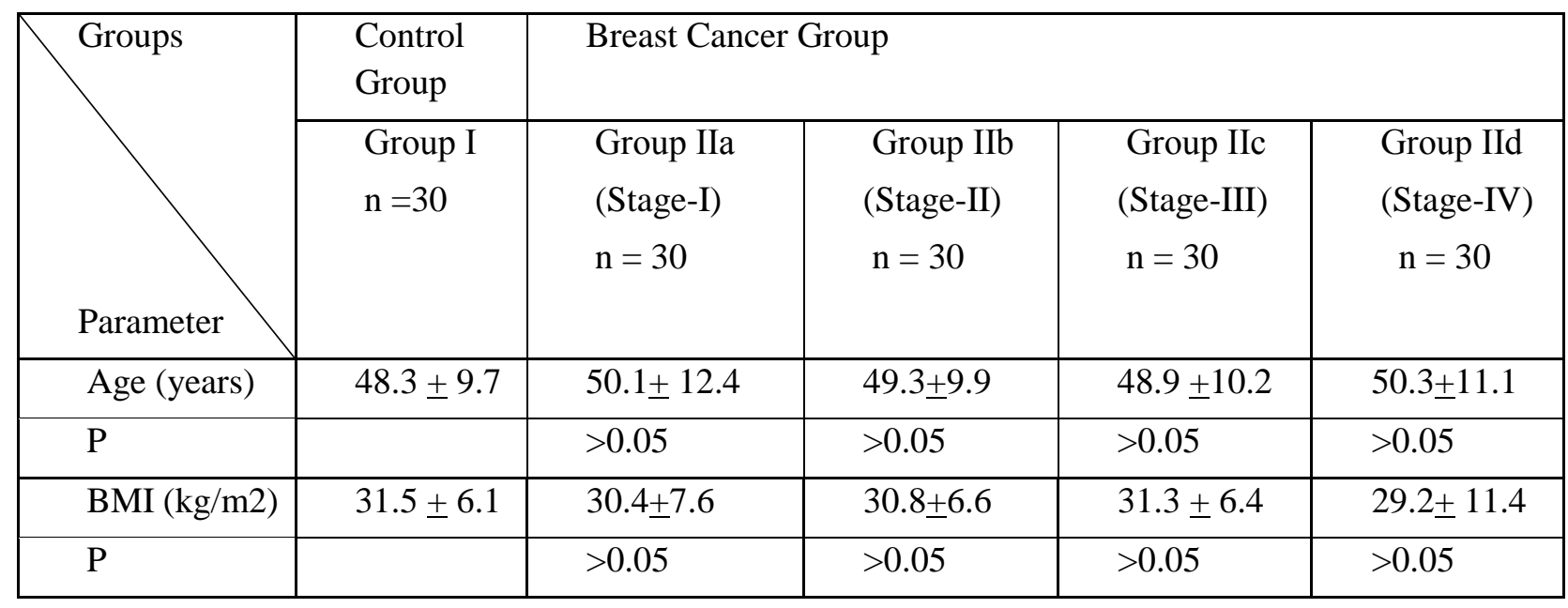

Histopathological type and tumor grade

The most prevalent histolopathological type of BC (99 cases; $82.5 \%$ ) was invasive ductal carcinoma (IDC). 9 cases $(7.5 \%)$ were invasive lobular carcinoma (ILC), 4 cases $(3.34 \%)$ were mucinous type, 3 cases $(2.5 \%)$ were medullary type, 3 cases $(2.5 \%)$ were malignant phyllodes tumor, and 2 cases $(1.66 \%)$ were poorly differentiated carcinoma. Regarding to the tumor grade, grade I tumors patients were $12(10 \%)$, grade II tumors patients were $79(65.8 \%)$ and grade III tumors were $29(24.2 \%)$. 


\section{NPI (table 2)}

Serum Omentin-1 levels

Step rise increase in the serum omentin-1 levels as the patients stage progress is evident in table 3 .

Table 2: The breast cancer patients prognosis according to the NPI values.

\begin{tabular}{|l|l|l|}
\hline Patients prognosis according to NPI & N & Percentage \\
\hline - Excellent prognosis. & 2 & $1.67 \%$ \\
\hline - Good prognosis. & 19 & $15.83 \%$ \\
\hline - Moderate prognosis. & 75 & $62.5 \%$ \\
\hline - Poor prognosis. & 24 & $20 \%$ \\
\hline$*$ Total & 120 & $100 \%$ \\
\hline
\end{tabular}

Table 3: the mean values of omentin-1 the groups of the study.

\begin{tabular}{|l|l|l|l|l|l|}
\hline Mean \pm SD & Control & Stage-I & Stage-II & Stage-III & Stage-IV \\
\hline \hline Omentin-1 & $98.4+$ & $161+49.6$ & $185.9+41$. & $\begin{array}{l}192.7+ \\
67.2\end{array}$ & $\begin{array}{l}230.2+ \\
43.6\end{array}$ \\
\hline $\mathrm{ng} / \mathrm{L})$ & 8.2 & & 8 & $<0.05$ & $<0.01$ \\
\hline $\mathrm{P}$ & & $<0.01$ & $>0.05$ & $<$ \\
\hline
\end{tabular}

Pearson correlation testing of the serum omentin-1 with the clinicopathological characteristics of the patients is shown in table 4 and figures $1,2,3 \& 4$ which revealed that omentin-1 levels were showing non-significant correlation with the histopathological grade, while significant correlation was noted with the patient age and high significant correlation was noted with tumor size, nodes status and NPI values.

Table 4: Correlations between the omentin-1 and different clinic-pathological parameters in the breast cancer patients.

\begin{tabular}{|l|c|c|}
\hline \multirow{2}{*}{ Variables } & \multicolumn{2}{|c|}{ Omentin-1 } \\
\cline { 2 - 3 } & $\mathrm{R}$ & $\mathrm{p}$ \\
\hline Age & 0.3 & $<0.05$ \\
\hline Tumor size & 0.37 & $<0.01$ \\
\hline Node status & 0.36 & $<0.01$ \\
\hline Tumor grade & 0.13 & $>0.05$ \\
\hline NPI values & 0.38 & $<0.01$ \\
\hline
\end{tabular}




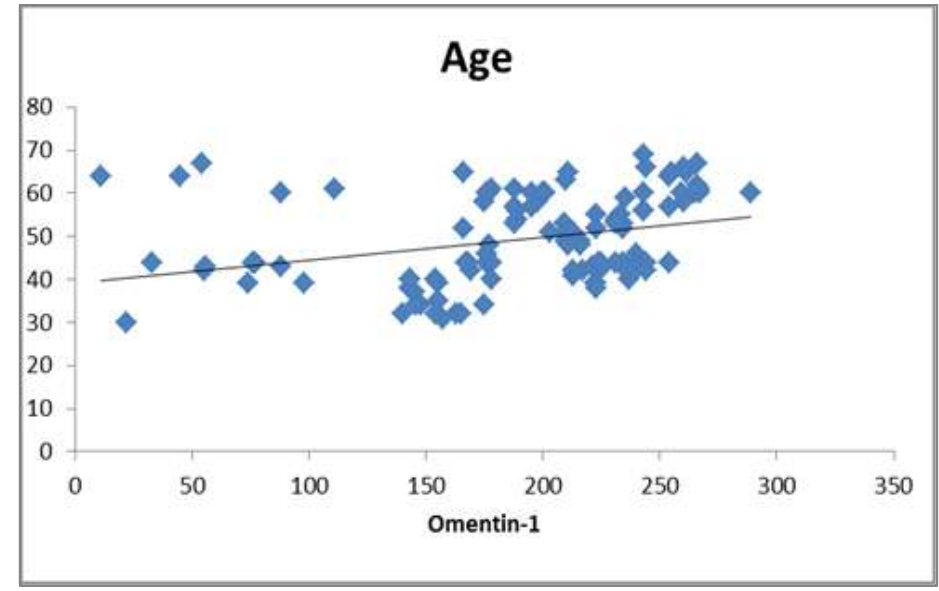

Figure 1: Serum omentin-1 level was significantly correlated to patient age.

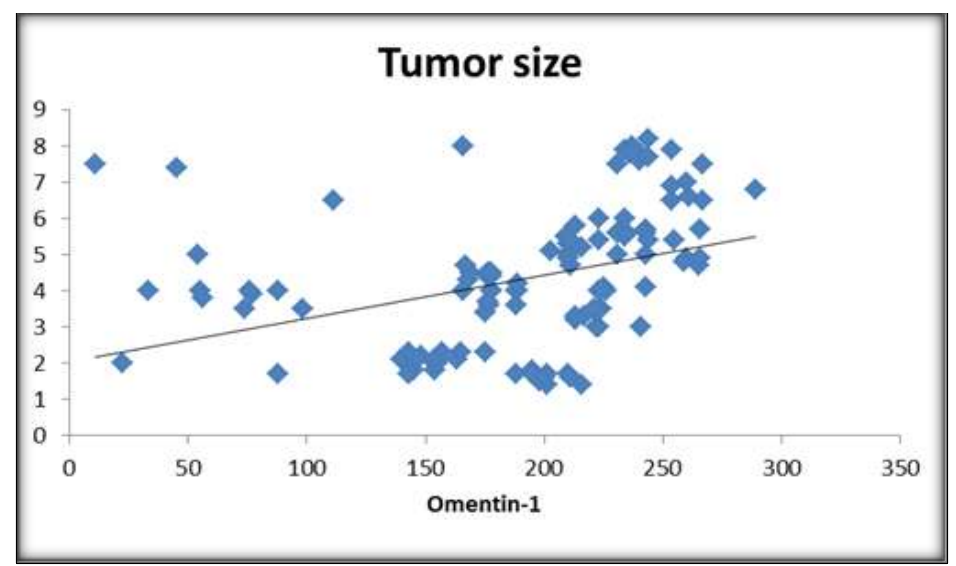

Figure 2: Serum omentin-1 level was highly significantly correlated to tumor size.

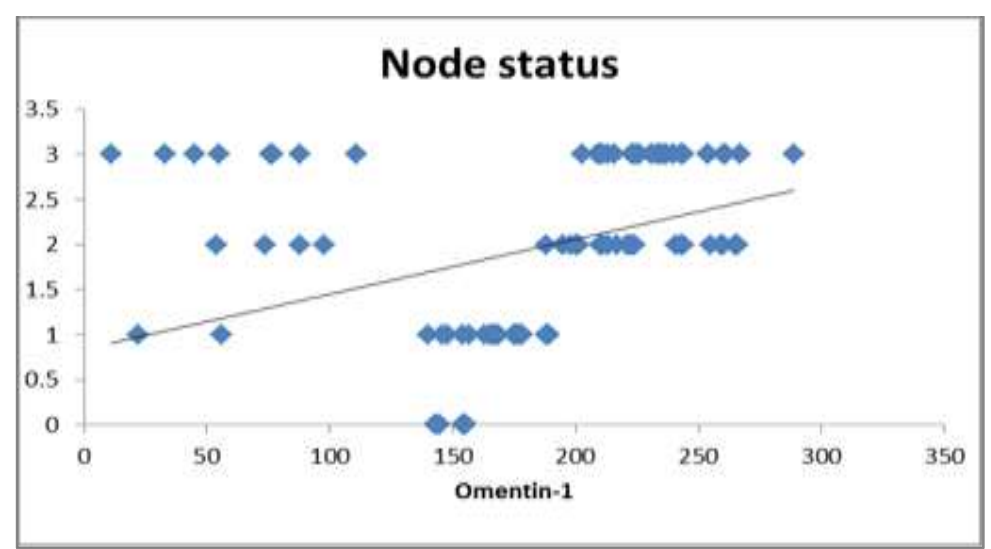

Figure 3: Serum omentin-1 level was highly significantly correlated to node status. 


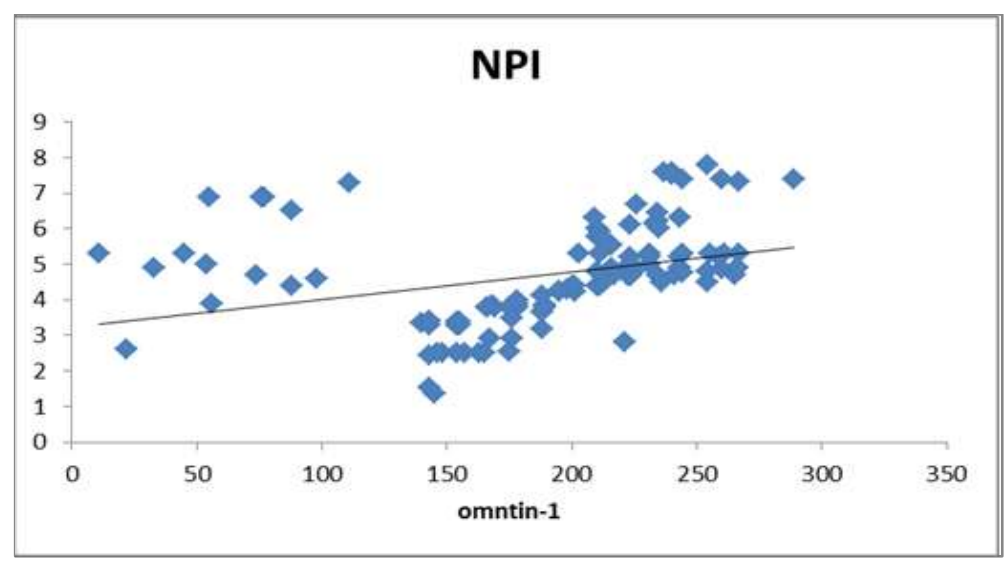

Figure 4: Serum omentin-1 level was highly significantly correlated to NPI.

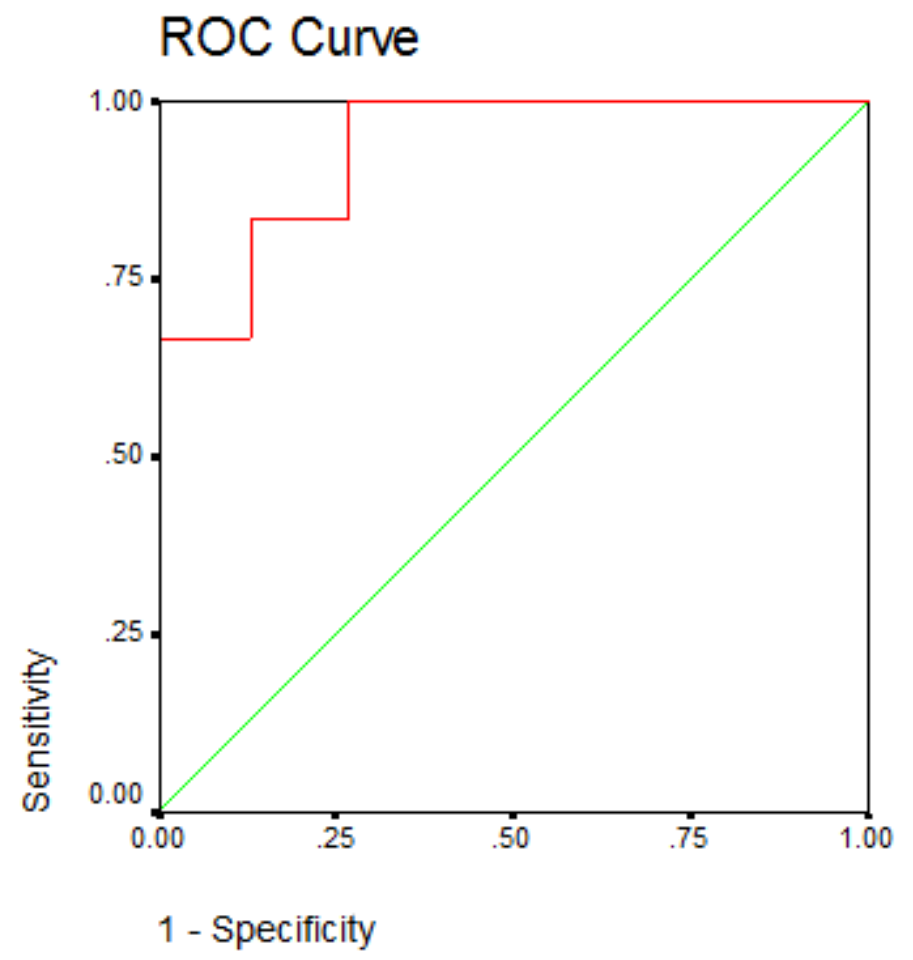

Figure 5: ROC curvesevaluationof Serumomentin-1 levels.

The serum omentin-1 cutoff value to differentiate healthy controls from breast cancer patients, was 128.3 $\mathrm{ng} / \mathrm{L}$ as found by the ROC curve evaluation as shown in Figure 5. The sensitivity and specificity of which were $82 \%$ and $78 \%$ respectively

\section{DISUSSION}

Most of the tumor markers are secreted by not only tumor cells, but also by the normal cells. However, their levels are much increased in cases of cancer [12].

Accordingly, the estimation of tumor markers levels in the serum is a valuable tool to assess the tumor activity. This is a little invasive and low cost and high effective method to determine the tumor activity, disease course, progression and proper management protocol. However, there are conflicting data about the 
reliability of tumor markers use in the breast cancer owing to some data about little sensitivity in the diagnosis of the early stages of the disease [13].

In this study, we aimed to assess the importance of evaluating serum levels of omentin-1 in the diagnosis as well as the prognosis of Egyptian female patients with breast cancer.

Omentin is an adipokine that is secreted mainly by visceral fat, it is $34 \mathrm{kDa}$ protein structure, known with its anti-inflammatory effect, it has two isoforms omentin-1 and omentin- 2. Omentin-1 is more abundant plasma form [14].

Conflicting results about omentin-1 effect on carcinogenesis were evident. Regarding to hepatocellular carcinoma, some studies showed the apoptotic effect of omentin-1 on cancer cells, while other studies showed reversed results as it concluded the inhibitory effect of omentin-1 on the apoptosis of cancer cells, therefore stimulating tumor activity and proliferation [15]. Researchers also reported controversial results about the association between omenti-1 levels and breast cancer [16].

Serum Omentin-1 biomarkers was studied in this study. There was significant elevation in the omentin-1 levels in the breast cancer patients compared to control group, and among the breast cancer patients subgroups, except for stage-I and stage-II groups, as they showed no significant difference. Also, after reassessing the difference in the serum omentin-1 levels among the different NPI categories. The serum levels of omentin-1 were significantly differing among the good, moderate and poor prognosis groups, while no significant difference was found between the excellent and the good prognosis groups. This study showed that serum levels of omentin-1 were significantly correlated with patients' age, tumor size, node status and the NPI status, whereas no significant correlation was noted with the tumor grade.

\section{CONCLUSION}

In this study, the serum omentin-1 cutoff value to differentiate healthy controls from breast cancer patients, was $128.3 \mathrm{ng} / \mathrm{L}$ as found by the ROC curve evaluation. The sensitivity and specificity of which were $82 \%$ and $78 \%$ respectively. This is comparable with the result found by Tahmasebpour et al, who found that the reliable cutoff value differentiating healthy subjects from breast cancer patients was $136.5 \mathrm{ng} / \mathrm{L}$, with concluded sensitivity and specificity of $63.64 \%$ and $89.02 \%$ respectively [17]. The results also of Nourbakhsh et al was in concordance with our results as the found that omentin-1 levels may be beneficial in the diagnosis and prognosis of invasive breast carcinoma [18].

In contrast with our results, some previous concluded that the serum omentin-1 levels are reduced in breast [18; 19]. This elucidates the need for more studies evaluating the role of omentin-1 breast cancer pathogenesis and prognosis and trying to explain the controversy in different studies results.

\section{CONCLUSION}

Our findings suggested that serum omentin- 1 could be diagnostic marker for early cases detection, and its serum levels revealed association with the breast cancer prognosis, as they are shown to have step rise increase as the disease stage get worse, ensuring its value as prognostic biomarker.

\section{REFERENCES}

[1] BALDASSARRE G AND BELLETTI B: Molecular biology of breast tumors and prognosis. F1000 Research journal. 13(1):5-9; 2016.

[2] ZAKARIA A, EL-KINAAI N, LOAY I, NASSAR H, DARWISH T, EL-DEFTAR M, ALLAM R AND NABIL H: Triple Negative Breast Cancer, Clinicopathologic Study of Egyptian Patients, NCI Experience. Medical Journal of Cairo University. 86(5):2747-2753; 2018. 
[3] TAO Z, SHI A AND LU C: BREAST CANCER: Epidemiology and Etiology. Cell Biochem Biophys. 72: 333-338; 2015.

[4] MICHAEL J DUFFY . Serum Tumor Markers in Breast Cancer: Are They of Clinical Value Clinical Chemistry, Volume 52, Issue 3 Pages 345-351, 2006.

[5] Edge S, Byrd D and Compton C: AJCC Cancer Staging Manual. $7^{\text {th }}$ ed. New York, NY: Springer. 13(7)347-376, 2010.

[6] YANG R.Z., LEE M.J., HU H., PRAY J., WU H.B., HANSEN B.C. Identification of omentin as a novel depot-specific adipokine in human adipose tissue: Possible role in modulating insulin action. Am. J. Physiol. Endocrinol. Metab;290: 2016.

[7] KAWASHIMA K, MAEDA K, SAIGO C, KITO Y, YOSHIDA K AND TAKEUCHI T: Adiponectin and intelectin-1: important adipokine players in obesity-related colorectal carcinogenesis. International journal of molecular sciences.;18(4):866-878, 2017.

[8] ALEKSANDROVA K, DI GIUSEPPE R, ISERMANN B, BIEMANN R, SCHULZE M, WITTENBECHER C AND PISCHON T: Circulating Omentin as a Novel Biomarker for Colorectal Cancer Risk: Data from the EPIC-Potsdam Cohort Study. Cancer research.;76(13):38623871, 2016.

[9] FRYCZKOWSKI M, BUŁDAK J, HEJMO T, KUKLA M AND ŻWIRSKA-KORCZALA K: Circulating levels of omentin, leptin, VEGF, and HGF and their clinical relevance with PSA marker in prostate cancer. Disease markers 3852401:9-21:2018.

[10] ZHANG Y.Y., ZHOU L.M. Omentin-1, a new adipokine, promotes apoptosis through regulating Sirt1-dependent p53 deacetylation in hepatocellular carcinoma cells. Eur. J. Pharmacol. 698:137144: 2013.

[11] GARNER J: "In the case of breast cancer, how can these be combined to give prognostic information?". Questions for the MRCS vivas. London: Arnold. p. 231: 2004.

[12] KABEL A: TUMOR MARKERS OF BREAST CANCER: New prospectives. Journal of Oncological Sciences. (3):5-11: 2017.

[13] BANEGAS P, Y. BIRD J, MORAROS S, KING S, PRAPSIRI B AND THOMPSON P: Breast cancer knowledge, attitudes, and early detection practices in United States-Mexico border Latinas, Journal Women's Health, 21 (1);(3):101-107; 2012.

[14] DE SOUZA BATISTA M, YANG Z, LEE J, GLYNN M, YU Z AND PRAY J: Omentin plasma levels and gene expression are decreased in obesity. Diabetes.;56(6):1655-1661; 2007 .

[15] YIN L, HUANG D, LIU X, WANG Y, LIU J AND LIU F: Omentin-1 effects on mesenchymal stem cells: Proliferation, apoptosis, and angiogenesis in vitro. Stem Cell Research \& Therapy. 8(1):224; 2017.

[16] NOURBAKHSH P, GANJI A, FARAHANI I, HOSSEINIAN R, YEGANEFARD F AND MIRZAEE R: Adipokine omentin-1: A diagnostic tool in breast cancer. International Journal of Basic Science in Medicine. 6(7):12-19; 2018.

[17] TAHMASEBPOUR N, HOSSEINPOUR-FEIZI A, ZIAMAJIDI N, POULADI N, MONTAZERI $\mathrm{V}$, FARHADIAN M AND ABBASALIPOURKABIR R: Association of Omentin-1 with Oxidative Stress and Clinical Significances in Patients with Breast Cancer. Advanced Pharmaceutical Bulletin, in press,available at $\quad$; https://apb.tbzmed.ac.ir/Files/Inpress/apb27961.pdf.:2009.

[18] UYETURK U, SARICI H, TEKCE BK, EROGLU M, KEMAHLI E, UYETURK U, ET AL. Serum omentin level in patients with prostate cancer. Medical Oncolology. 31(4):923-931; 2014.

[19] SHEN D, ZHANG L, CHE H, ZHANG Y, YANG C AND ZHOU J: Circulating levels of adipocytokine omentin1 in patients with renal cell cancer. ,Cytokine. 77:50-55; 2016. 